\title{
Fisher Information of Landau States and Relative Information against the Lowest Level ${ }^{+}$
}

\author{
Takuya Yamano \\ Kanagawa University, Yokohama, Japan \\ + Presented at the Entropy 2021: The Scientific Tool of the 21st Century, 5-7 May 2021; Available online: \\ https://sciforum.net/conference/Entropy2021/.
}

Published: 5 May 2021

An electron in a constant magnetic field has the energy levels known as the Landau levels. One can obtain the corresponding radial wave function in cylindrical polar coordinates (e.g., textbook of Landau \& Lifshitz). This system is not explored so far in terms of information-theoretical point of view. We here focus on Fisher information associated with these Landau states specified by the two quantum numbers. Fisher information provides a useful measure of the electronic structure in quantum systems such as hydrogen-like atoms [1,2] and molecules under Morse potentials [3]. We numerically evaluate the generalized Laguerre polynomials contained in the radial wave functions. We report that Fisher information increases linearly with the quantum number $n$ that specifies energy levels, but decreases monotonically with the quantum number $\mathrm{m}$ (i.e., the index of the generalized Laguerre polynomial).

Also, we present relative Fisher information of the Landau states by setting the lowest Landau state as a reference density. The analytical form is just $4 n$, which does not depend on the other quantum number $\mathrm{m}$.

\section{References}

1. Yamano, T. Relative Fisher information of hydrogen-like atoms. Chem. Phys. Lett. 2018, 691, 196-198.

2. Yamano, T. Fisher information of radial wavefunctions for relativistic hydrogenic atoms. Chem. Phys. Lett. 2019, 731, 136618.

3. Yamano, T. Relative Fisher information for Morse potential and isotropic quantum oscillators. J. Phys. Commun. 2018, 2, 085018.

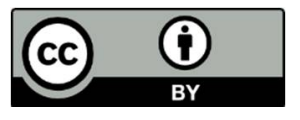

(C) 2021 by the author. Licensee MDPI, Basel, Switzerland. This article is an open access article distributed under the terms and conditions of the Creative Commons Attribution (CC BY) license (http://creativecommons.org/licenses/by/4.0/). 